\title{
Editorial
}

\section{Modeling consumer behavior}

Journal of Revenue and Pricing Management (2011) 10, 399-400. doi:10.1057/rpm.2011.23

Consumer choice is a theory of microeconomics that relates preferences to consumer demand curves. The link between personal preferences, consumption and the demand curve is one of the most complex relations in economics. Implicitly, economists assume that anything purchased will be consumed, unless the purchase is for a productive activity. From a revenue management perspective, it is assumed that consumer demand for each of the fare products is completely independent of the controls being applied by the seller. However, this is not the case in reality. So what is the revenue management community doing about this to improve the accuracy, relevance and develop the science?

A good demand forecast should be at the heart of every revenue management model. Yet most demand models focus on product demand and do not incorporate customer choice behavior under offered alternative says Haensal and Koole who use the ideas of customer choice sets to model the customer's buying behavior. A customer choice set is a set of product classes representing the buying preferences and choice decisions of a certain customer group. In this article the authors present a demand estimation method for these choice sets. The authors highlight that previous research papers have shown that there is a huge need in revenue management for a move from product-based demand to customer choice demand. The authors are convinced that there method will be helpful in achieving this goal. Even though using maximum likelihood to approximate an exponential curve from data points is in general ill conditioned, the resulting demand curves fit the original ones reasonably well. In addition, using the excerpt information to control the future booking process increases the resulting revenue, as well as the accuracy of future estimation.

Kunnumkal and Topaloglu propose a randomized linear program to obtain bid prices for the network revenue management problem with customer choice behavior. This development proceeds in two stages. First formulate a mixed integer program that can be viewed as a deterministic approximation to the network revenue management problem, then use the optimal solution to the mixed integer program to formulate a linear program that can be easily randomized. The results from a practitioner perspective conclude that this approach is appealing as it can be easily implemented by using commercially available software and it can provide substantial improvements over the standard choice-based deterministic linear program.

\section{OTHER PAPERS}

Advance selling has become a universal practice in managing perishable product, Zhaung and Li examine a revenue management problem with random demands from an advance market and a spot market when the revenue manager is risk averse with an a temporal utility function. The authors demonstrate that risk-averse managers value more of immediate revenue than potentially higher yet uncertain future revenue, hence tend to allocate more capacity for the advance market to hedge against the risk originated from the uncertainty in the spot market demand.

Kumar and Prevost evaluate whether an outside health and wellness services operation partnering with a business organization can 
influence and improve the health attitudes and behaviors of its employees and increase productivity, which results in reduced health-care costs and increased revenues. The results find organizations realize significant gains in productivity and savings in health insurance costs that far outweigh the cost of instituting health and wellness programs for employees through a service provider. Employees participating in such programs also pay lower health insurance premiums. Further, analysis of data gathered from the two surveys showed employees greatly value participation in health and wellness program. Such program positively impacts attitude and behavior change in employees toward health and wellness.

\section{THE FUTURE}

Vinod's futures article provides an overview of the origins and significance of the airline frequent flyer programs for competitive advantage and subsequent adoption across various industry travel verticals. The future of successful loyalty programs will be contingent on leveraging data augmented with advanced analytics to gain valuable insight to enhance the customer engagement process, retain and reward profitable customers.

\section{AND FINALLY ...}

Phillips reviews the eleventh annual INFORMS Revenue Management and Pricing Section Conference was held at Columbia University in New York City in June 2011, a conference jointly hosted by the Columbia University Business School and Department of Industrial Engineering and Operations Research.

Ian Yeoman

Editor

Follow the Journal on twitter@RevManJournal 